\title{
SPATIOTEMPORAL PATTERNS OF THE CALIFORNIA SPOTTED OWL'S TERRITORIAL VOCALIZATIONS
}

CONNOR M. WOOD, STEPHANIE M. SCHMIDT, and M. ZACHARIAH PEERY, University of Wisconsin-Madison, Department of Forest and Wildlife Ecology, Russell Laboratories Room 237, 1630 Linden Drive, Madison, Wisconsin 53706; cwood9@wisc.edu

ABSTRACT: Emerging bioacoustic technology allows researchers to passively record animal vocalizations to study population dynamics at relatively broad spatial scales. Spatiotemporal patterns in vocalization behavior free from direct human influence, which occurs during traditional vocal-lure surveys, may also yield novel behavioral insights. We used passively recorded audio data to examine such patterns in the California Spotted Owl (Strix occidentalis occidentalis) across the northern Sierra Nevada. We assessed temporal patterns in the initiation of bouts of four-note calls and the number of such calls, and we used generalized linear mixed models to test whether environmental factors influenced bout-initiation time, bout duration, and the number of calls per unit time. We found that (1) Spotted Owls were most vocally active within three hours of sunset, (2) bouts of calling were longer with fewer calls per unit time in open and young forest, (3) those bouts were concentrated in the middle of the night, and (4) the frequency of occurrence and duration of bouts of territorial vocal activity was reduced in montane riparian forest. These patterns suggest that Spotted Owls may engage in territorial defense in marginal habitat (open and young forest) and minimize their vocal activity in key foraging habitat (montane riparian forest). This application of acoustic monitoring data to behavioral ecology illustrates the broad applicability of the underlying audio data and its potential to yield novel ecological insights.

Vocalization is essential to the life history of many animal species (Schmidt et al. 2010). This is particularly true for birds, many of which employ a repertoire of vocalizations and sonations to communicate with individuals and groups of their own and other species (Webster and Podos 2018). Birds' extensive use of sound has allowed researchers to survey otherwise cryptic species by broadcasting or imitating their vocalizations (Forsman 1983, Dewey et al. 2003, Conway and Gibbs 2005, Savage et al. 2010, Braun De Torrez et al. 2017). However, conspecific and heterospecific vocalizations can influence the focal species' behavior (Crozier et al. 2006, Van Lanen et al. 2011), which can preclude the collection of unbiased behavioral data during vocal-lure surveys. The advent of low-cost acoustic-recording hardware and efficient signal-processing software may help resolve this issue and thus lead to novel insights into behavioral ecology (Shonfield and Bayne 2017).

Animal vocalizations that are identified with passively recorded audio represent the individual's response to its environment without direct human influence, allowing researchers to draw much stronger inferences about its behavior. For example, diel patterns in vocal activity could provide insight into a bird's roosting habitat because a bird is necessarily closer to its roost site at the start and end of its activity periods. The duration of bouts of vocalization as well as the relative density of vocalizations per bout could provide clues to the intent of the vocalizations. For example, long bouts of 
calling that contain relatively few calls per unit time would be consistent with territorial maintenance or defense (e.g., Ganey 1990).

We explored the spatiotemporal variation in the vocalization patterns of the California Spotted Owl (Strix occidentalis occidentalis), the subject of a continuing acoustic-monitoring program on a regional scale (Wood et al. 2019). Specifically, we focused on the four-note call, which is used by the owls for territorial defense and, to a lesser extent, for intrapair communication (Forsman et al. 1984, Ganey 1990). It is also used as an indicator of site occupancy in the monitoring program. Our objectives were to describe nightly patterns of vocal activity, to model the time of initiation and duration of bouts of calling, and to model the number of calls per unit time in response to environmental variables. If vocalizations are not uniformly distributed in time (e.g., vocalizations are concentrated in certain times), the efficiency of acoustic-monitoring programs could be improved by focusing on periods of consistently high vocal activity. A non-uniform distribution of vocalizations in space would suggest that owls are modifying their behavior in response to the habitat around them; such variation could provide insight into their natural history. Analyzing vocalization patterns in this and other ways can improve our understanding of Spotted Owl behavior and help increase the efficiency of acoustic-monitoring programs.

\section{METHODS}

\section{Field and Bioacoustic Methods}

Our study took place from 7 May to 8 June 2018 in the Lassen and Plumas national forests in the northern Sierra Nevada, California. Three hundred and nineteen survey sites, each consisting of a 400-ha hexagon (the approximate size of a Spotted Owl territory in this area), were distributed randomly but noncontiguously across the study area to promote statistical independence among sites. Within each site, we deployed two or three autonomous recording units (ARUs, Swift Recorder, Cornell Lab of Ornithology Bioacoustics Research Program) without knowledge of whether an owl occupied the site. We placed the ARUs $\geq 500 \mathrm{~m}$ apart because in situ testing indicated that they had an effective sampling distance, or listening radius, of $\sim 250 \mathrm{~m}$. They were programmed to record continuously from 20:00 to 06:00 Pacific Daylight Time; during our study sunset was at approximately 20:15, sunrise at approximately 05:40. These surveys were part of a larger, continuing program of acoustic monitoring in the area (see Wood et al. 2019).

To identify Spotted Owl vocalizations, we scanned the resulting audio data with a moving window template detector (Raven 2.0, Cornell Lab of Ornithology, Bioacoustics Research Program), which identifies instances in the input data that match user-defined targets, in this case high-quality examples of the Spotted Owl's four-note call above a given threshold. We used a relatively high matching threshold with a goal of detecting at least one four-note call in any given bout of calling (see Wood et al. 2019 for further details). To count the number of calls, we reviewed all sound signals identified by the template detector as potential Spotted Owl calls. On the basis 
of 14 bouts of intrapair vocalizations, we classified vocalizations as male if the lower bound of the notes was $\leq 625 \mathrm{~Hz}$ or as female if the lower bound of the notes was $\geq 631 \mathrm{~Hz}$. Next, we reviewed the audio before and after all calls identified by the template detector and subsequently confirmed as of the Spotted Owl in order to manually count four-note calls that had been recorded but were below the detector's matching threshold. We reviewed the recordings until 10 minutes had elapsed without any Spotted Owl vocalization (including contact calls or other nontarget vocalizations, which were not included in our counts) because the average duration of a bout of Spotted Owl calling is 10 minutes (Ganey 1990). We then defined a bout of calling as a sequence of four-note calls in which no calls were greater than 10 minutes apart. Because the rate of detection of the Spotted Owl in these surveys is $<1$ (Wood et al. 2019), it is likely that some occupied sites were excluded from the data, but imposing a temporal limit on our dataset ensured that the calls we included were all made at similar times in the season and thus in comparable behavioral and ecological contexts.

\section{Analytical Methods}

First, we qualitatively described temporal patterns of the Spotted Owl's four-note vocalization within a night. Next, we examined relationships between three aspects of the species' vocalization behavior and remotely sensed data representing environmental variables. To do so, we buffered all the ARUs' locations by $250 \mathrm{~m}$, the distance defined by their listening radius (see above) and calculated the proportion of that area represented by various land-cover types. We classified forest as open (canopy cover $<40 \%$ ), young (canopy cover $\geq 40 \%$ and quadratic mean diameter of tree trunks $<31 \mathrm{~cm}$ ), medium (canopy cover $\geq 40 \%$ and quadratic mean diameter of tree trunks $31-61 \mathrm{~cm}$ ), or old (canopy cover $\geq 40 \%$ and quadratic mean diameter of tree trunks $\geq 61 \mathrm{~cm}$ ) on the basis of gradient-nearest-neighbor data $(30 \mathrm{~m}$ resolution) from the LEMMA lab at Oregon State University (lemma.forestry. oregonstate.edu/data/structure-maps). We also calculated the proportion composed of montane riparian forest (30 m resolution; www.mrlc.gov/ national-land-cover-database-nlcd-2016) and calculated the average slope (5 m resolution; digital elevation model from the U.S. Geological Survey).

Our response variables were (1) the initiation time of a bout of calling in hours from the average time of sunset/sunrise (whichever was closer) while the ARUs were recording, (2) the duration of a bout of calling, and (3) the residuals of the linear relationship between bout duration and the number of calls in the bout ("duration-count residuals"). Bout duration and the number of calls per bout were highly correlated ( $r=0.77$, Figure 1). A positive value of the bout's duration-count residual indicates a greater-thanexpected number of calls per unit time, whereas a negative value indicates a lower-than-expected number. Using these three response variables and the remotely sensed covariates, we constructed three sets of generalized linear mixed models. We tested the models' fit that included the ARU and survey site as random effects to account for the many units that recorded multiple bouts of calling and for non-independence among ARUs deployed at the same site. However, models were overfit (indicated by singularity, i.e., perfect correlation between explanatory variables), so we removed the 


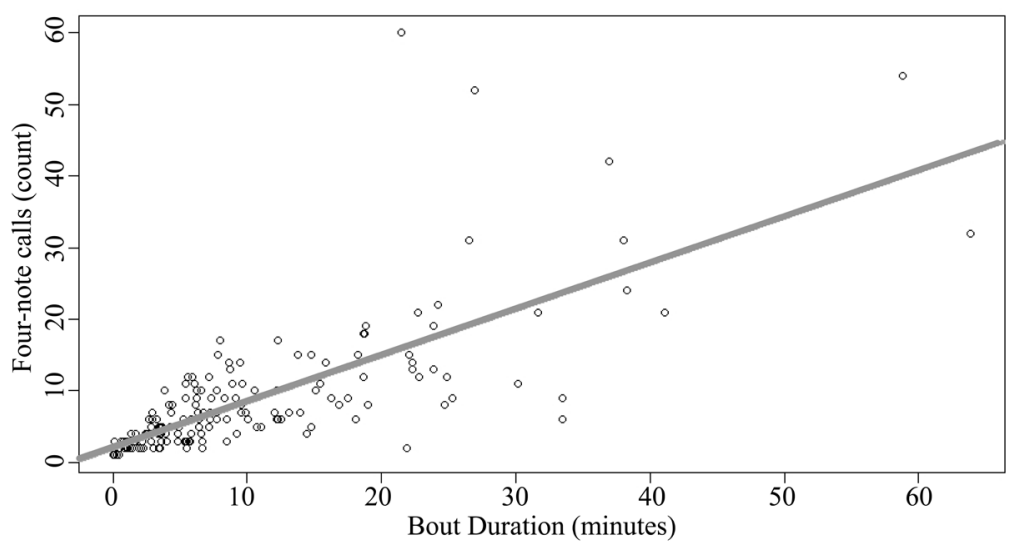

Figure 1. The duration of a bout of Spotted Owl calling was highly correlated with the number of four-note calls in that bout $(r=0.77)$. Points above the line [count $=$ $1.94+0.64 \times$ duration (minutes); adjusted $R^{2}=0.59 ; F=313.6, \mathrm{df}=214, p<$ 0.0001 ] have positive residuals and therefore represent a bout of calling with more calls per unit time than expected.

higher-order random effect (i.e., site) (Zuur et al. 2009). For each model, we calculated Akaike's information criterion with a correction for small sample size $\left(\mathrm{AIC}_{c}\right)$, ranked models in each of the three sets (corresponding with our three response variables), and considered models with a difference from the best model $(\Delta \mathrm{AIC})$ of $<2$ to be supported by the data (Burnham and Anderson 2010). We used program R (R Core Development Team 2014) and packages xlsx (Dragulescu and Arendt 2018), lme4 (Bates et al. 2019), and MuMIn (Bartón 2015) for these analyses.

\section{RESULTS}

Seventy-one recorders deployed within 45 of 319 survey sites detected the four-noted vocalization of the Spotted Owl, for a total of 1531 calls in 216 bouts. The number of bouts and the total number of calls peaked between one and two hours after sunset (21:00 and 22:00; Figure 2), and $53 \%$ of both bouts and calls were recorded within three hours of sunset (20:00-23:00). Of 839 vocalizations identified by the template detector (i.e., were above our matching threshold), $91.8 \%$ were produced by males; 93.1\% of the 216 bouts contained male vocalizations only, $4.2 \%$ contained both male and female vocalizations, and $2.8 \%$ contained female vocalizations only. The paucity of female vocalizations precluded the use of an owl's sex or its status as single or paired as a covariate in subsequent analyses.

Bouts of vocalization initiated further in time from sunrise or sunset were associated with less montane riparian forest $\left(\beta_{\mathrm{Mt} . \mathrm{Rp} .}=-0.79, \mathrm{SE}=1.60\right.$; Table 1 ), and greater amounts of old forest ( $\beta$ Old $=0.55, \mathrm{SE}=1.45$ ) and open forest ( $\beta$ Open $=0.36, \mathrm{SE}=1.09$ ). However, the high sampling error associated with these estimates suggests that relationships between time 

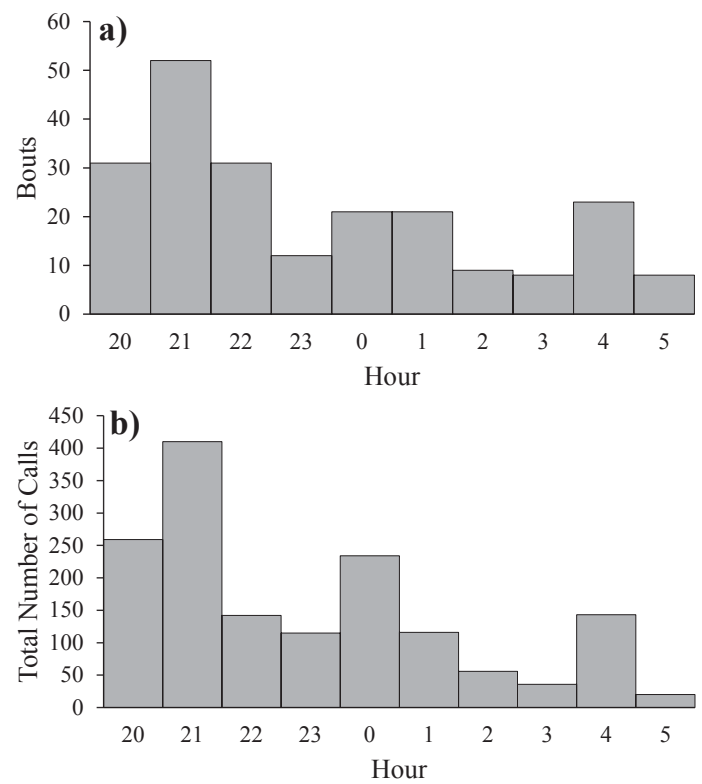

Figure 2. Times at which Spotted Owls initiated bouts of four-note calls (a), and the total number of calls recorded. We defined a bout as a group of calls isolated from all other Spotted Owl vocalizations by at least 10 minutes. Calls were passively recorded from 7 May to 8 June 2018 under a standardized protocol; recording units were deployed without prior knowledge of the owl's occupancy of the site.

of initiation of a bout of calling and habitat variables may be influenced by other, unmeasured factors.

The duration of bouts of four-note calling decreased with the amount of old, montane riparian, and medium forest $\left(\beta\right.$ old $=-18.8, \mathrm{SE}=11.04 ; \beta_{\mathrm{Mt}}$. Rp. $=-13.92, \mathrm{SE}=12.62 ; \beta_{\text {Medium }}=-7.70, \mathrm{SE}=4.48$; Table 1 ; Figure $3 \mathrm{a}-\mathrm{e}$ ) and increased with the amount of young and open forest ( $\beta$ Young $=$ 9.46 (4.71); $\beta$ Open $=9.46$ (4.71); Table 1).

The ARUs recorded fewer calls per unit time than expected (i.e., lower duration-count residuals) in areas with more open forest ( $\beta_{\text {Open }}=-6.25, \mathrm{SE}$ $=3.83$; Figure $3 \mathrm{f}$ ). No other models of the number of calls per unit time contained informative parameters (Table 1; Arnold 2010).

\section{DISCUSSION}

The Spotted Owl's four-note call is essential to several aspects of its natural history (Forsman et al. 1984, Ganey 1990) and is used to determine site occupancy in a landscape-scale acoustic-monitoring program in the northern Sierra Nevada (Wood et al. 2019). The temporal patterns we documented suggest that most territorial vocalization is concentrated within 
Table 1 Generalized Linear Mixed Models of Patterns of Spotted Owl Vocalizing with Respect to Habitat Features ${ }^{a}$

\begin{tabular}{lccc}
\hline Response variable and covariate & $\beta(\mathrm{SE})^{b}$ & $\Delta \mathrm{AIC}_{c}$ & $w$ \\
\hline Hours to daylight & & & \\
Mt. riparian & $-0.79(1.60)$ & 0.00 & 0.26 \\
Old forest & $0.55(1.45)$ & 0.30 & 0.22 \\
Open forest & $0.36(1.09)$ & 0.90 & 0.16 \\
Null & NA & 0.94 & 0.16 \\
Young forest & $-0.46(0.61)$ & 1.62 & 0.11 \\
Medium forest & $0.14(0.56)$ & 2.28 & 0.08 \\
Slope & $-0.02(0.02)$ & 7.65 & 0.01 \\
Duration & & & \\
Old forest & $-18.8(11.04)$ & 0.00 & 0.31 \\
Young forest & $9.46(4.71)$ & 0.57 & 0.24 \\
Mt. riparian & $-13.92(12.62)$ & 1.30 & 0.16 \\
Open forest & $11.12(8.54)$ & 1.60 & 0.14 \\
Medium forest & $-7.7(4.48)$ & 1.64 & 0.14 \\
Null & $\mathrm{NA}$ & 7.35 & 0.01 \\
Slope & $-0.15(0.15)$ & 10.38 & 0.00 \\
Duration-count residuals & & & \\
Open forest & $-6.25(3.83)$ & 0.00 & 0.28 \\
Null & $\mathrm{NA}$ & 0.61 & 0.21 \\
Young forest & $2.46(1.96)$ & 1.08 & 0.16 \\
Slope & $-0.06(0.06)$ & 1.80 & 0.11 \\
Mt. riparian & 2.16 & 2.51 & 0.08 \\
Old forest & $-1.81(5.03)$ & 2.53 & 0.08 \\
Medium forest & $-0.41(1.81)$ & 2.61 & 0.08 \\
\hline
\end{tabular}

${ }^{a}$ For each response variable, the models are listed by increasing distance in $\mathrm{AIC}_{c}$ from the best model and in decreasing order of weight $(w)$. All models contained recording unit as a random effect. ${ }^{b}$ Estimates of $\beta$ for models of various forest types can be compared because all are on the same scale (proportion of area in a $250-\mathrm{m}$ radius around the location of each recorder comprising a given habitat type).

approximately three hours of sunset, consistent with the patterns reported by Ganey (1990) on the basis of vocal-lure surveys. In areas of younger and more open forest, generally considered lower-quality habitat, territorial vocalization (i.e., bouts of four-note calls) tended to be farther in time from daylight, longer, and less frequent. Vocalizing was less frequent and briefer in montane riparian forest. Finally, bouts of territorial vocalizations were relatively short in old forest. Interspecific interactions, which may not be uncommon (Wood et al. 2019), may also be influencing these patterns, but our results nonetheless represent a nuanced picture of Spotted Owl vocalization behavior that is consistent with what is known about the species' natural history.

The tendency of bouts of calling to be longer in open and young forest, and the relatively low number of vocalizations per unit time in open forest, suggest that male owls, which produced the vast majority of the vocalizations, are engaging in territorial defense or maintenance in those areas (Ganey 

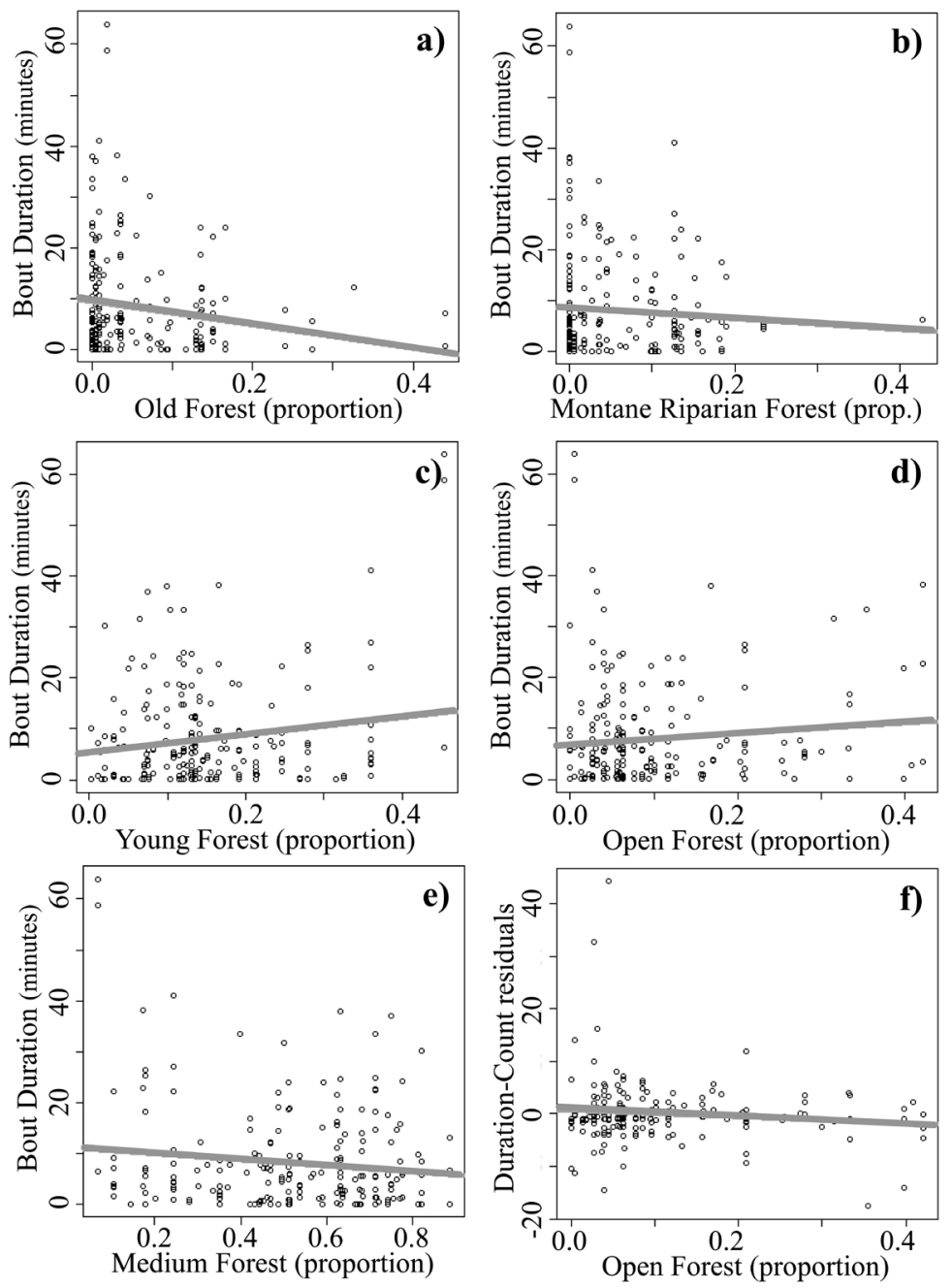

Figure 3. Relationships between the best-supported forest-cover variables $\left(\Delta \mathrm{AIC}_{\mathrm{c}}<\right.$ 2; Table 1) and (a-e) duration of bouts of calling and ( $\mathrm{f}$ ) the relative density of calls per unit time. Forest cover was assessed with remotely sensed data and is measured as the proportion of the area around a given recording unit.

1990). The positive relationship between open forest and calls' time to daylight further suggests that the owls in open forest may be calling away from roost sites and outside core areas, possibly along territory margins. Open forest (canopy cover $<40 \%$ ) and young forest (trees of low quadratic mean diameter) are not preferred Spotted Owl habitat (Moen and Gutiérrez 
1997, North et al. 2017, Jones et al. 2018, Hobart et al. 2019), though stands of open forest with high basal area of hardwoods contribute to Spotted Owl reproduction (Hobart et al. 2019) and some stands of young forest are selected for foraging (Atuo et al. 2019). Collectively, this behavior suggests that Spotted Owls dedicate time to active maintenance of the boundaries of their territories, despite relatively routine movements that often encompass multiple territories (Berigan et al. 2018, Blakey et al. 2019). GPS tags with audio-recording capabilities would be necessary to test this hypothesis.

For roosting and nesting, Spotted Owls preferentially select stands with large trees and closed canopy (Call et al. 1992, Moen and Gutiérrez 1997), and in our study area the amount of such habitat in a territory is positively associated with sustained occupancy over multiple seasons (Jones et al. 2018). Shorter bouts of four-note calls associated with more extensive old forest could represent intrapair communication. If we had included other vocalizations, such as contact calls, some bouts of vocalizations would have been longer and contained many more vocalizations. Indeed, we observed several instances in which a male Spotted Owl produced four-note vocalizations and a female responded with contact calls: in our dataset such behavior would be represented as a bout of calling by the male only. Including contact calls and other vocalizations, however, would have precluded many comparisons because the biological meanings of contact calls and four-note calls, for example, are different (Forsman et al. 1984).

Our finding that montane riparian forests were associated with fewer bouts of calling overall, particularly in the middle of the night (further in time from daylight), may imply that Spotted Owls are aware of the behavior and perception of their prey. In our study area, montane riparian zones are important nesting and foraging areas for Humboldt's flying squirrel (Glaucomys oregonensis) (Meyer et al. 2005, 2007), and flying squirrels are important prey of the Spotted Owl (Zabel et al. 1995). Therefore, vocalizing in montane riparian forest could alert potential prey to the owls' presence and thus decrease hunting success. This hypothesis could be tested by equipping Spotted Owls with high-resolution GPS tags.

The concentration of four-note calls in the three hours after sunset suggests that acoustic monitoring of the Spotted Owl could be effective without recording through the entire night. For example, programming the ARUs to shut themselves off from 2:00 to 4:00 each night would expedite data processing (e.g., copying data from memory cards and compressing audio files for long-term storage) and the review of template-detector results. Over the course of a season, this modification would save approximately 3.2 terabytes of data under current specifications (Wood et al. 2019). Such reductions can lead to substantial time and cost savings over the life of a long-term monitoring program, and, because vocal activity is low during that period, few potential detections are likely to be missed (Figure 2a). Indeed, if the only vocalization detected at a site is recorded in the middle of the night, it may be the product of a transient rather than territorial individual (Berigan et al. 2018). Tags that record both sound and location by GPS could again prove useful if the resulting data identify times of night during which Spotted Owls are more or less likely to vocalize closer or farther from their territory centers. 
Our findings may help refine acoustic-monitoring programs designed for the Spotted Owl and represent an application of such data to the field of behavioral ecology. The inclusion of additional vocalizations, both intra- and interspecific, could clarify the relationships we identified further. Importantly, our findings can yield testable hypotheses. For example, future analyses might examine whether contact calls are more closely associated with the Spotted Owl's preferred habitat than are four-note calls, or compare highresolution GPS data on foraging locations to remotely sensed habitat data. The habitat-mediated patterns of the Spotted Owl's vocalizing illustrate the various ecological constraints that the necessities of territorial defense, intrapair communication, and foraging impose. In such a context, the absence of vocalization can be just as informative as an abundance of calls. This application of acoustic-monitoring data to behavioral ecology illustrates the broad utility of the underlying audio data, and the potential of such data to yield novel ecological insights.

\section{ACKNOWLEDGMENTS}

We thank Michaela Gustafson for leadership in the field and extensive bioacoustic work, Clark Hollenberg, Wynter Lim, Caitlyn Powell, and Kevin Wood for their efforts in the field, Rodney Siegel and an anonymous reviewer whose input improved this manuscript, and Region 5 of the U.S. Forest Service, the California Department of Fish and Wildlife, and the University of Wisconsin-Madison Department of Forest and Wildlife Ecology for funding.

\section{LITERATURE CITED}

Arnold, T. W. 2010. Uninformative parameters and model selection using Akaike's information criterion. J. Wildlife Mgmt. 74:1175-1178; doi 10.1111/j.19372817.2010.tb01236.x.

Atuo, F. A., Roberts, K., Whitmore, S., Dotters, B. P., Raphael, M. G., Sawyer, S. C., Keane, J. J., Gutiérrez, R. J., and Peery, M. Z. 2019. Resource selection by GPS-tagged California Spotted Owls in mixed-ownership forests. Forest Ecol. Mgmt. 433:295-304; doi 10.1016/j.foreco.2018.11.011.

Bartón, K. 2015. Multi-model inference. Comprehensive R Archive Network; https:// cran.r-project.org/web/packages/MuMIn/index.html.

Bates, D. Maechler, M., Bolker, B., Walker, S., Christensen, R., Singmann, H., Dai, B. Scheipl, F., Groothendieck, G., Green, P., and Fox, J. 2019. Linear mixed-effects models using "Eigen" and S4. Comprehensive R Archive Network; www.cran.r-project.org/web/packages/lme4/index.html.

Berigan, W. J., Jones, G. M., Whitmore, S. A., Gutiérrez, R. J., and Peery, M. Z. 2018. Cryptic wide-ranging movements lead to upwardly biased occupancy in a territorial species. J. Appl. Ecol. 56:470-480; doi 10.1111/1365-2664.13265.

Blakey, R. V., Siegel, R. B., Webb, E. B., Dillingham, C. P., Bauer, R. L., Johnson, M., and Kesler, D. C. 2019. Space use, forays, and habitat selection by California Spotted Owls (Strix occidentalis occidentalis) during the breeding season: New insights from high resolution GPS tracking. Forest Ecol. Mgmt. 432:912-922; doi 10.1016/j.foreco.2018.10.017.

Braun De Torrez, E. C., Samoray, S. T., Silas, K. A., Wallrichs, M. A., Gumbert, M. W., Ober, H. K., and McCleery, R. A. 2017. Acoustic lure allows for capture of a high-flying, endangered bat. Wildlife Soc. Bull. 41:322-328; doi 10.1002/ wsb.778. 
Burnham, K. P., and Anderson, D. R. 2010. Model Selection and Multimodel Inference: A Practical Information-Theoretic Approach. Springer, New York.

Call, D. R., Gutiérrez, R. J., and Verner, J. 1992. Foraging habitat and homerange characteristics of California Spotted Owls in the Sierra Nevada. Condor 94:880-888; doi 10.2307/1369285.

Conway, C. J., and Gibbs, J. P. 2005. Effectiveness of call-broadcast surveys for monitoring marsh birds. Auk 122:26-35; doi 10.1642/0004-8038(2005)122[0026:EO CSFM]2.0.CO;2.

Crozier, M. L., Seamans, M. E., Gutiérrez, R. J., Loschl, P. J., Horn, R. B., Sovern, S. G., and Forsman, E. D. 2006. Does the presence of Barred Owls suppress the calling behavior of Spotted Owls? Condor 108:760-769; doi 10.1650/0010-5422(2006)108[760:DTPOBO]2.0.CO;2.

Dewey, S. R., Kennedy, P. L., and Stephens, R. M. 2003. Are dawn vocalization surveys effective for monitoring goshawk nest-area occupancy? J. Wildlife Mgmt. 67:390-397; doi 10.2307/3802779.

Dragulescu, A. A., and Arendt, C. 2018. Xlsx. Comprehensive R Archive Network; www.cran.r-project.org/web/packages/xlsx/index.html.

Forsman, E. D. 1983. Methods and materials, for locating and studying Spotted Owls. U.S. Forest Service Pacific Northwest Forest and Range Experiment Station Gen. Tech. Rep. PNW-162.

Forsman, E. D., Meslow, E. C., and Wight, H. M. 1984. Distribution and biology of the Spotted Owl in Oregon. Wildlife Monogr. 87:3-64; doi 10.2737/PNWGTR-162.

Ganey, J. L. 1990. Calling behavior of Spotted Owls in northern Arizona. Condor 92:485-490; doi 10.2307/1368245.

Hobart, B. K., Roberts, K. N., Dotters, B. P., Berigan, W. J., Whitmore, S. A., Raphael, M. G., Keane, J. J., Gutiérrez, R. J., and Peery, M. Z. 2019. Site occupancy and reproductive dynamics of California Spotted Owls in a mixedownership landscape. Forest Ecol. Mgmt. 437:188-200; doi 10.1016/j. foreco.2019.01.028.

Jones, G. M., Keane, J. J., Gutiérrez, R. J., and Peery, M. Z. 2018. Declining old-forest species as a legacy of large trees lost. Diversity and Distributions 24:341-351; doi 10.1111/ddi.12682.

Meyer, M. D., Kelt, D. A., and North, M. P. 2005. Nest trees of northern flying squirrels in the Sierra Nevada. J. Mammal. 86:275-280.

Meyer, M. D., Kelt, D. A., and North, M. P. 2007. Microhabitat associations of northern flying squirrels in burned and thinned forest stands of the Sierra Nevada. Am. Midland Nat. 157:202-211; doi 10.1644/BEH-110.1.

Moen, C. A., and Gutiérrez, R. J. 1997. California Spotted Owl habitat selection in the central Sierra Nevada. J. Wildlife Mgmt. 61:1281-1287; doi $10.2307 / 3802127$.

North, M. P., Kane, J. T., Kane, V. R., Asner, G. P., Berigan, W., Churchill, D. J., Conway, S., Gutiérrez, R. J., Jeronimo, S., Keane, J., Koltunov, A., Mark, T., Moskal, M., Munton, T., Peery, M. Z., Ramirez, C., Sollmann, R., White, A., and Whitmore, S. 2017. Cover of tall trees best predicts California Spotted Owl habitat. Forest Ecol.Mgmt. 405:166-178; doi 10.1016/j.foreco.2017.09.019.

R Core Development Team. 2014. R: A language and environment for statistical computing. R Foundation for Statistical Computing, Vienna, Austria.

Savage, A., Thomas, L., Leighty, K. A., Soto, L. H., and Medina, F. S. 2010. Novel survey method finds dramatic decline of wild cotton-top tamarin population. Nature Comm. 1:30; doi 10.1038/ncomms1030.

Schmidt, K. A., Dall, S. R. X., and Gils, J. A. V. 2010. The ecology of information: An overview on the ecological significance of making informed decisions. Oikos 119:304-316; doi 10.1111/j.1600-0706.2009.17573.x. 
Shonfield, J., and Bayne, E. M. 2017. Autonomous recording units in avian ecological research: Current use and future applications. Avian Cons. Ecol. 12(1), article 14; doi 10.5751/ACE-00974-120114.

Van Lanen, N. J., Franklin, A. B., Huyvaert, K. P., Reiser, R. F., and Carlson, P. C. 2011. Who hits and hoots at whom? Potential for interference competition between Barred and Northern Spotted Owls. Biol. Cons. 144:2194-2201; doi 10.1016/j.biocon.2011.05.011.

Webster, M. S., and Podos, J. 2018. Acoustic Communication, in Ornithology (M. L. Morrison, A. D. Rodewald, G. Voelker, M. R. Colón, and J. F. Prather, eds.), pp. 409-436. Johns Hopkins Univ. Press, Baltimore.

Wood, C. M., Gutiérrez, R. J., and Peery, M. Z. 2019. Acoustic monitoring reveals a diverse forest owl community, illustrating its potential for basic and applied ecology. Ecology, in press; doi 10.1002/ecy.2764.

Wood, C. M., Popescu, V. D., Klinck, H., Keane, J. J., Gutiérrez, R. J., Sawyer, S. C., and Peery. M. Z. 2019. Detecting small changes in populations at landscape scales: A bioacoustic site-occupancy framework. Ecol. Indicators 98:492-507; doi 10.1016/j.ecolind.2018.11.018.

Zabel, C. J., McKelvey, K., and Ward, J. P. Jr. 1995. Influence of primary prey on home-range size and habitat-use patterns of Northern Spotted Owls (Strix occidentalis caurina). Can. J. Zool. 73:433-439; doi 10.1139/z95-049.

Zuur, A., Ieno, E. N., Walker, N., Saveliev, A. A., and Smith, G. M. 2009. Mixed Effects Models and Extensions in Ecology with R. Springer-Verlag, New York; doi 10.1007/978-0-387-87458-6.

Accepted 8 July 2019

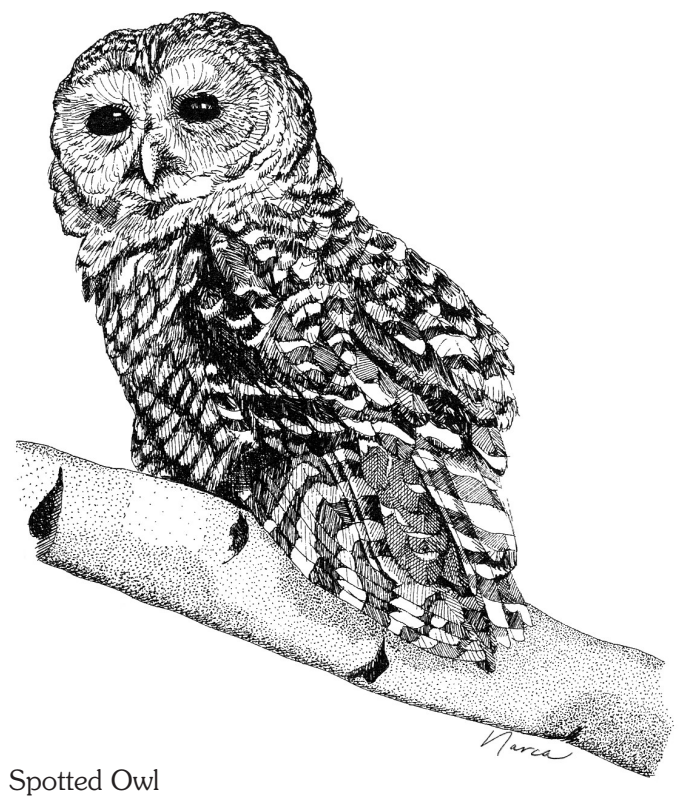

Sketch by Narca Moore-Craig 